\title{
Effect of Kinetic Resistance Training and Technique on Special Strength Level and Effective Kinematic Variables in Instep Kick for Soccer Juniors
}

\author{
Amr Ali Shady \\ Sport Training Department, Faculty of Sport Education, Mansoura University, Mansoura, Egypt \\ E-mail: dr_ashady2004@yahoo.com
}

Received: 11-11- 2015

Accepted: 19-12- 2015

Published: 31-01- 2016

doi:10.7575/aiac.ijkss.v.4n.1p.33

URL: http://dx.doi.org/10.7575/aiac.ijkss.v.4n.1p.33

\begin{abstract}
Background: Training with resistance is considered the essential and complementary part of players' preparation period during training season through developing different aspects. Objective: This study aims to investigate the Effect of Kinetic resistance training and technique on special power level and effective kinematic variables in instep kick for soccer juniors. Methodology: 20 junior soccer players (age: $17.54 \pm 0.5$ years, body mass: $69.05 \mathrm{~kg}$, height: $170 \mathrm{~cm}$, training age $=7.7$ years) participated in this study were randomly assigned into two groups experimental $(\mathrm{n}=10)$ trained with kinetic resistance training program and technique and control $(n=10)$ trained with traditional soccer training program only where, the experimental approach was used. Results: The kinetic resistance trainings and technique positively affected the special power level, kicking accuracy and time and effective kinematic variables in instep kick. Conclusion: The researcher recommended that coaches should give attention to special strength developing and to be an essential part of the training program through kinetic resistance trainings and technique. Also coaches should depend on the kinematic variables affecting performance for detecting the improvement level in performance as a result of kinetic resistance trainings and technique.
\end{abstract}

Keywords: kinetic resistance, technique, special strength, kinematic, Instep Kick

\section{Introduction}

Soccer has been affected by the scientific development in sports and physical education to develop players' abilities and achievements through taking care of special physical preparation devoted to increasing functional efficiency of vital organs and developing physical and skillful characteristics discriminating soccer player. Training with resistance is considered the essential and complementary part of players preparation period during training season through developing different aspects. The scientific researches and studies indicated the improvement in young players physical fitness level following the resistance training programs right instruction and steps (Roberts \& Weider, 1994).

Muscular strength in its different shapes including explosive strength, maximum strength and rate of force development plays a major role in enhancing such skills performance (Cabri et al., 1988). And hence for soccer player to perform different shapes of skill performances during the game, some physical abilities should be available. Where these physical abilities type, quantity and timing differentiate based on each skill singularity and type (Keshek \& Elbosaty , 2000).

The success in kinetic skill performance depends on player ability in employing physical components especially the muscular strength during skill performance. Different players have high levels of muscular strength that appears in muscular strength tests but couldn't be applied in skill performance as it requires the ability of good kinetic correlation and movement right transmission. The good co-operation of the kinetic transmission process of the exerted strength from the trunk to the thigh then leg then ankle works in an open reaction chain starting from trunk to ankle (Noguchi et, al., 2012). The muscular strength in the upper and lower part of the body moves through a set of muscles groups participating in soccer kicking skill. Thus during the design of soccer players special strength developing training programs especially juniors, central muscles strength working in physical activity should be developed and the exercises should be similar to the performance nature not only the shape but also the speed of each part of the body and in the same muscular working direction(Schmitz,2003).

Through this study the researcher defined the Kinetic Resistance as a resistance based on rubber bands (as shown in Figure 1) where the player exerts muscular contractions with shape and kinetic path similar to the soccer kinetic performances type. The instep kick is one of the most needed skills in soccer, constituting a basic element of the game when a faster and more powerful ball needs to be generated (Inoue et.al., 2012). So the existence of information about the needed characteristics through different skills performance became an essential for coaches where the technological 
development in motion analysis field has revealed the interlaced relations between body parts motions through performing skills that can be obtained by tracking and analyzing player motion during the performance stages of these skills.
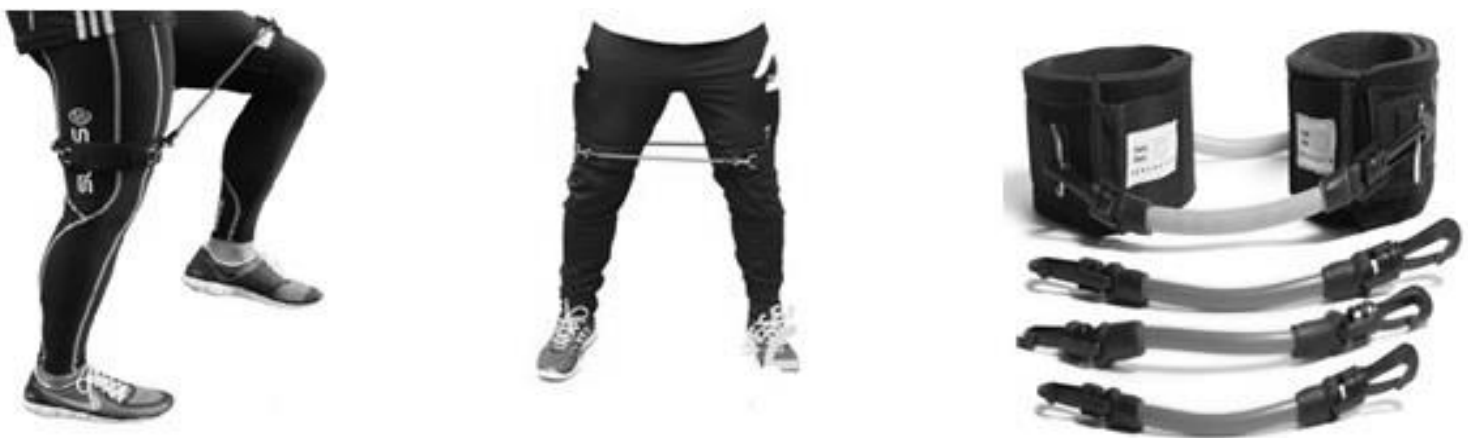

Figure 1. The rubber calibration bands used in the kinetic resistance training program (Resistance Band, 2015)

Kinematic analysis and kinematic variables extraction for the instep kick is one of the most preferred methods for detecting the extent to which players have benefit from the gained strength levels whereas the speed change in leg parts including hip, leg or ankle is a strong indicator to evaluate the player ability to benefit of the gained strength levels he has (Reilly \& Williams, 2003).

The researcher indicates that detecting the characteristics of the kinetic performance during training and modifying it according to the skill kinetic performance goal is one of the substantial tasks for the soccer training programs success. Hence the more similarities between exercise and actual kinetic performance, the more peculiarity the exercise and its effectiveness for enhancing skill kinetic performance increases. Selecting the appropriate training method depends on diagnosing and accurately characterizing the skill kinetic performance to determine the role of muscular strength as an essential physical variable in this performance, and since the special strength training style starts from the skill kinetic performance characteristics as a primary base in selecting training method and building exercises to be used in soccer dedicated training.

The researcher noticed that the inability of many players on employing the muscular strength, they have in kinetic performance specially at soccer kicking since most players have high strength levels but cannot employ this strength during kinetic performance. Through the researcher acquaintance on several previous studies and scientific references in sport training as general and soccer field specially, it was clear to him the paucity of these studies that handled the effect of kinetic resistance trainings on kicking skill, however, these studies have clarified the importance of special strength developing on improving kicking skill. Hence appears the importance of this study as one of the experimental studies that proposes a new method for developing soccer juniors kicking skill and providing the necessary information needed by soccer coaches.

From all of the above the researcher in this study aims to investigate the effectiveness of kinetic resistance training and technique on the special strength level, instep kick performance accuracy and time and kinematic variables affecting the instep kick of soccer juniors in the experimental group compared to the control group.

And in order to go through this study the researcher assumed that

i. There are significant differences between the experimental group pre-test and post-test measurements of the special strength, soccer kicking performance level and kinematic variables affecting the soccer juniors instep kick for the post-test.

ii. There are significant differences between the control group pre-test and post-test measurements of the special strength, soccer kicking performance level and kinematic variables affecting the soccer juniors instep kick for the post-test.

iii. There are significant differences between the control and experimental groups in the post test measurement of the special strength, soccer kicking performance level and kinematic variables affecting the soccer juniors instep kick for the experimental group post-test.

In next section the research methodology and sample are described in detail indication the research variables and the training program foundations including training units time and number of repetitions.

\section{Methods}

\subsection{Research Methodology}

The researcher utilized the experimental methodology through the experimental design of two groups experimental and control to achieve the aims of research and hypnoses. 


\subsection{Research Sample}

Twenty soccer juniors were selected (age: $17.54 \pm 0.5$ years, body mass: $69.05 \mathrm{~kg}$, height: $170 \mathrm{~cm}$, training age $=7.7$ years) and randomly assigned into two groups experimental $(\mathrm{n}=10)$ and control $(\mathrm{n}=10)$.

\subsection{Research variables}

- Special Strength : was evaluated using (three consecutive springs speed - three right hops speed- three left hops speed- vertical jump) tests knowing that speed $=$ distance/ time.

- Instep Kick: was evaluated using (kicking accuracy - kicking time) tests.

- Kinematic variables affecting the instep kick: the following variables were extracted (Angular displacement $(\theta)$ - Linear displacement $\left(S^{\mathrm{R}}\right)$ - Angular velocity $(\omega)$ - Linear velocity $\left(\mathrm{V}^{\mathrm{R}}\right)$ - Linear acceleration $\left.\left(\mathrm{a}^{\mathrm{R}}\right)\right)$.

The researcher utilized high-speed video camera (250 frame / sec.) and the "Motion Track" movement analysis program to extract the kinematic variables though determining the appropriate time moments to detect theses variables that were prepared to be statistically processed.

\subsection{The Training Program Foundations}

The scientific foundations and regulations of the training program were determined according to (Perrin, 1993; Evans, 1997; Moran \& George, 2000) as following:

- Integrated and proper warm up that includes general flexibility

- The exercise should be performed during the full range of the exercise movement.

- The special strength training program should start with the muscular strength establishment using the general and inclusive exercises for all body muscles and that was considered by the researcher through the body weight exercises and the low intensity polymeric exercises for the different muscles sets during the general preparation stage (two weeks long).

- The exercise should be similar to the game skill that tack place during completion and has been trained on.

- The training load components should proportioned ( intensity - size - rest ) according to what that scientists have reported as following:

○ Load Intensity: 50-80\% of the maximum limit of the individual level.

○ Load Size: (8-12) repetitions.

○ Sets count : (3:4) sets

$\circ$ Rest between repetitions: (1-3) from performance time.

○ Rest between sets: (3-4) minutes.

$\circ$ Number of training units: 3 training units per week.

$\circ$ Duration of the program: 8 weeks

- The content of the training program is executed after a good warm up in other words in the main part of the training unit and then the rest of the training unit is continued.

- The training duration in each training unit ranges from 35:45 minutes.

OStatic flexibility exercises are performance during rest.

\section{Results}

Table (1) indicates the existence of significant difference between the pre-test and post-test in special strength and instep kicking time and accuracy of the experimental group for the post-test.

Table 1. Differences between pre-test and post-test of Special Strength and instep kick of the experimental group

\begin{tabular}{|c|c|c|c|c|c|c|c|}
\hline \multirow{2}{*}{$\begin{array}{c}\mathrm{n}=10 \\
\text { Variables }\end{array}$} & \multirow{2}{*}{ 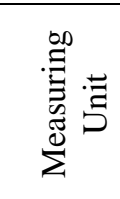 } & \multicolumn{2}{|c|}{ Pre-test } & \multicolumn{2}{|c|}{ Post test } & \multirow{2}{*}{ 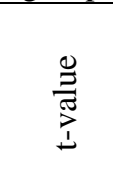 } & \multirow{2}{*}{ 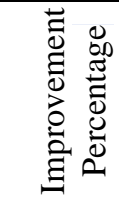 } \\
\hline & & Mean & Std. D & Mean & Std. D & & \\
\hline three consecutive springs speed test & $\mathrm{m} / \mathrm{sec}$ & 2.504 & 0.05 & 2.799 & 0.02 & 17.75 & $\% 11.78$ \\
\hline three right hops speed test & $\mathrm{m} / \mathrm{sec}$ & 2.72 & 0.02 & 2.985 & 0.09 & 8.85 & $\% 9.74$ \\
\hline three left hops speed test & $\mathrm{m} / \mathrm{sec}$ & 2.702 & 0.02 & 3.01 & 0.07 & 16.19 & $\% 11.40$ \\
\hline vertical jump test & $\mathrm{c} \mathrm{m}$ & 47.5 & 2.64 & 73.7 & 2.67 & 56.14 & $\% 55.16$ \\
\hline kicking accuracy & Degree & 2.77 & 0.03 & 4.385 & 0.02 & 137.31 & $\% 58.30$ \\
\hline kicking time & $\mathrm{Sec}$ & 0.38 & 0.02 & 0.28 & 0.02 & 16.218 & $\% 26.70$ \\
\hline
\end{tabular}

T. spreadsheet at $0.05=1.833$ 


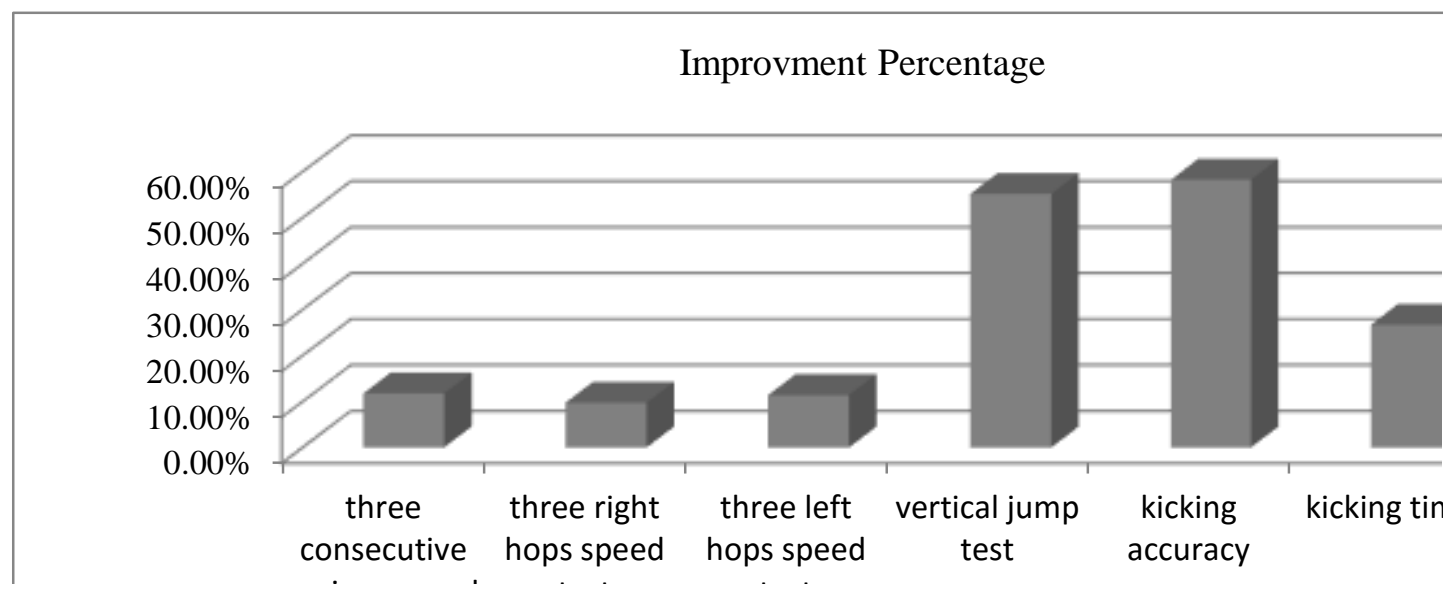

Figure 2. Improving percentage between pre-test and post-test of special strength and instep kick variables of the experimental group

Table 2. Differences between pre-test and post-test of the kinematic variables of the experimental group

\begin{tabular}{|c|c|c|c|c|c|c|c|c|}
\hline \multirow{2}{*}{$\mathrm{n}=10$} & \multirow{2}{*}{ Variables } & \multirow{2}{*}{ skill Stages } & \multicolumn{2}{|c|}{ Pre-test } & \multicolumn{2}{|c|}{ Post test } & \multirow{2}{*}{ t-value } & \multirow{2}{*}{$\begin{array}{c}\text { Improvement } \\
\text { Percentage }\end{array}$} \\
\hline & & & Mean & $\begin{array}{c}\text { Std. } \\
\text { D }\end{array}$ & Mean & Std. D & & \\
\hline \multirow{15}{*}{$\begin{array}{l}\text { Hip } \\
\text { Joint }\end{array}$} & \multirow{3}{*}{$\begin{array}{c}\text { Angular } \\
\text { displacement }(\theta)\end{array}$} & backswing & 162.5 & 9.85 & 175.7 & 1.34 & 4.43 & $\% 8.12$ \\
\hline & & Impact & 125.06 & 1.95 & 131.13 & 0.74 & 8.98 & $\% 4.85$ \\
\hline & & Follow-up & 116.14 & 1.32 & 118.64 & 0.13 & 6.36 & $\% 2.15$ \\
\hline & \multirow{3}{*}{ Linear $\underset{\left(S^{\mathrm{k}}\right)}{\operatorname{displacement}}$} & backswing & 0.271 & 0.02 & 0.356 & 0.02 & 9.60 & $\% 31.37$ \\
\hline & & Impact & 0.209 & 0.02 & 0.269 & 0.01 & 8.78 & $\% 28.71$ \\
\hline & & Follow-up & 0.28 & 0.02 & 0.371 & 0.01 & 11.24 & $\% 32.50$ \\
\hline & \multirow{3}{*}{ Angular velocity $(\omega)$} & backswing & 11.81 & 1.44 & 18.95 & 0.01 & 15.78 & $\% 60.42$ \\
\hline & & Impact & 0.411 & 0.03 & 0.62 & 0.01 & 22.32 & $\% 50.85$ \\
\hline & & Follow-up & 7.197 & 1.30 & 9.221 & 0.01 & 4.93 & $\% 28.12$ \\
\hline & \multirow{3}{*}{ Linear velocity $\left(\mathrm{V}^{\mathrm{R}}\right)$} & backswing & 1.286 & 0.06 & 1.828 & 0.01 & 23.52 & $\% 42.15$ \\
\hline & & Impact & 1.291 & 0.06 & 1.821 & 0.01 & 24.13 & $\% 41.05$ \\
\hline & & Follow-up & 0.725 & 0.05 & 1.109 & 0.15 & 9.30 & $\% 52.97$ \\
\hline & \multirow{3}{*}{$\begin{array}{c}\text { Linear acceleration } \\
\left(\mathrm{a}^{\mathrm{R}}\right)\end{array}$} & backswing & 12.059 & 0.47 & 13.942 & 0.02 & 12.25 & $\% 15.61$ \\
\hline & & Impact & 10.996 & 0.84 & 13.757 & 0.15 & 10.02 & $\% 25.11$ \\
\hline & & Follow-up & 12.732 & 1.19 & 15.8 & 0.09 & 8.22 & $\% 24.10$ \\
\hline \multirow{15}{*}{$\begin{array}{l}\text { Knee } \\
\text { Joint }\end{array}$} & \multirow{3}{*}{$\begin{array}{c}\text { Angular } \\
\text { displacement }(\theta)\end{array}$} & backswing & 65.9 & 1.10 & 68.4 & 1.07 & 5.51 & $\% 3.79$ \\
\hline & & Impact & 110.6 & 0.84 & 112.8 & 0.79 & 4.71 & $\% 1.99$ \\
\hline & & Follow-up & 171.8 & 4.10 & 178.5 & 1.08 & 4.63 & $\% 3.90$ \\
\hline & \multirow{3}{*}{$\begin{array}{c}\text { Linear displacement } \\
\left(\mathrm{S}^{\mathrm{R}}\right)\end{array}$} & backswing & 0.426 & 0.03 & 0.318 & 0.02 & $8.26-$ & $\% 25.35$ \\
\hline & & Impact & 1.996 & 0.04 & 2.03 & 0.04 & 2.25 & $\% 1.70$ \\
\hline & & Follow-up & 2.625 & 0.08 & 2.725 & 0.02 & 3.28 & $\% 3.81$ \\
\hline & \multirow{3}{*}{ Angular velocity $(\omega)$} & backswing & 1.854 & 0.03 & 2.461 & 0.07 & 22.14 & $\% 32.74$ \\
\hline & & Impact & 14.06 & 1.07 & 19.195 & 0.63 & 12.06 & $\% 36.52$ \\
\hline & & Follow-up & 0.286 & 0.02 & 0.347 & 0.01 & 9.04 & $\% 21.33$ \\
\hline & \multirow{3}{*}{ Linear velocity $\left(\mathrm{V}^{\mathrm{R}}\right)$} & backswing & 4.521 & 0.30 & 5.638 & 0.15 & 9.60 & $\% 24.71$ \\
\hline & & Impact & 1.305 & 0.30 & 2.638 & 0.20 & 11.17 & $\% 102.15$ \\
\hline & & Follow-up & 2.75 & 0.18 & 3.223 & 0.03 & 8.39 & $\% 17.20$ \\
\hline & \multirow{3}{*}{$\begin{array}{l}\text { Linear acceleration } \\
\left(\mathrm{a}^{\mathrm{R}}\right)\end{array}$} & backswing & 52.59 & 1.28 & 55.505 & 0.28 & 6.69 & $\% 5.54$ \\
\hline & & Impact & 57.6 & 0.97 & 61.1 & 1.10 & 8.71 & $\% 6.08$ \\
\hline & & Follow-up & 32.7 & 0.95 & 35.5 & 1.43 & 9.63 & $\% 8.56$ \\
\hline \multirow{15}{*}{$\begin{array}{l}\text { Ankle } \\
\text { Joint }\end{array}$} & \multirow{3}{*}{$\begin{array}{c}\text { Angular } \\
\text { displacement }(\theta)\end{array}$} & backswing & 151.6 & 1.26 & 152.5 & 0.97 & 1.96 & $\% 0.59$ \\
\hline & & Impact & 128 & 1.49 & 133.6 & 1.26 & 7.07 & $\% 4.38$ \\
\hline & & Follow-up & 138.9 & 1.10 & 139.6 & 0.84 & 2.68 & $\% 0.50$ \\
\hline & & backswing & 0.384 & 0.01 & 0.423 & 0.01 & 10.30 & $\% 10.16$ \\
\hline & Linear displacement & Impact & 0.414 & 0.01 & 0.46 & 0.02 & 9.66 & $\% 11.11$ \\
\hline & & Follow-up & 0.609 & 0.01 & 0.686 & 0.02 & 14.88 & $\% 12.64$ \\
\hline & & backswing & 3.552 & 0.02 & 4.527 & 0.76 & 4.02 & $\% 27.45$ \\
\hline & Angular velocity $(\omega)$ & Impact & 2.981 & 0.67 & 4.693 & 0.07 & 7.52 & $\% 57.43$ \\
\hline & & Follow-up & 1.527 & 0.24 & 1.953 & 0.02 & 5.37 & $\% 27.90$ \\
\hline & & backswing & 5.263 & 0.30 & 6.485 & 0.18 & 9.81 & $\% 23.22$ \\
\hline & Linear velocity $\left(\mathrm{V}^{\mathrm{R}}\right)$ & Impact & 5.831 & 1.19 & 8.959 & 0.02 & 8.24 & $\% 53.64$ \\
\hline & & Follow-up & 2.609 & 0.28 & 3.839 & 0.06 & 15.02 & $\% 47.14$ \\
\hline & & backswing & 24.085 & 0.83 & 28.7 & 0.82 & 12.87 & $\% 19.16$ \\
\hline & Linear acceleration & Impact & 124.8 & 2.44 & 130.5 & 0.71 & 6.75 & $\% 4.57$ \\
\hline & & Follow-up & 52 & 0.82 & 54.9 & 0.88 & 7.65 & $\% 5.58$ \\
\hline
\end{tabular}

T. spreadsheet at $0.05=1.833$ 
Table (2) indicates the existence of significant difference between the pre-test and post-test in kinematic variables (Angular displacement $(\theta)$-Linear displacement (SR)- Angular velocity ( $\omega)$ - Linear velocity (VR)-Linear acceleration $(\mathrm{aR}))$ of the experimental group for the post-test.

Table 3. Differences between pre-test and post-test in the Special Strength and instep kick of the control group

\begin{tabular}{|c|c|c|c|c|c|c|c|}
\hline \multirow{2}{*}{$\begin{array}{c}\mathrm{n}=10 \\
\text { Variables }\end{array}$} & \multirow{2}{*}{ 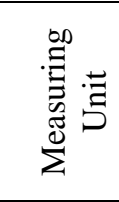 } & \multicolumn{2}{|c|}{ Pre-test } & \multicolumn{2}{|c|}{ Post test } & \multirow{2}{*}{ 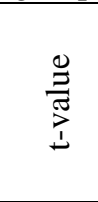 } & \multirow{2}{*}{ 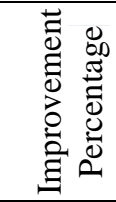 } \\
\hline & & Mean & Std. D & Mean & Std. D & & \\
\hline three consecutive springs speed test & $\mathrm{m} / \mathrm{sec}$ & 2.48 & 0.05 & 2.65 & 0.02 & 7.62 & $\% 6.59$ \\
\hline three right hops speed test & $\mathrm{m} / \mathrm{sec}$ & 2.70 & 0.02 & 2.82 & 0.03 & 13.41 & $\% 4.36$ \\
\hline three left hops speed test & $\mathrm{m} / \mathrm{sec}$ & 2.69 & 0.01 & 2.79 & 0.02 & 18.22 & $\% 4.01$ \\
\hline vertical jump test & $\mathrm{c} \mathrm{m}$ & 46 & 2.40 & 58.1 & 5.59 & 6.448 & $\% 26.30$ \\
\hline kicking accuracy & Degree & 2.78 & 0.03 & 3.42 & 0.20 & 9.48 & $\% 23.31$ \\
\hline kicking time & sec & 0.37 & 0.01 & 0.34 & 0.01 & 5.77 & $\% 8.47$ \\
\hline
\end{tabular}

T. spreadsheet at $0.05=1.833$

Table (3) indicates the existence of significant difference between the pre-test and post-test in special strength and instep kicking time and accuracy of the control group for the post-test.

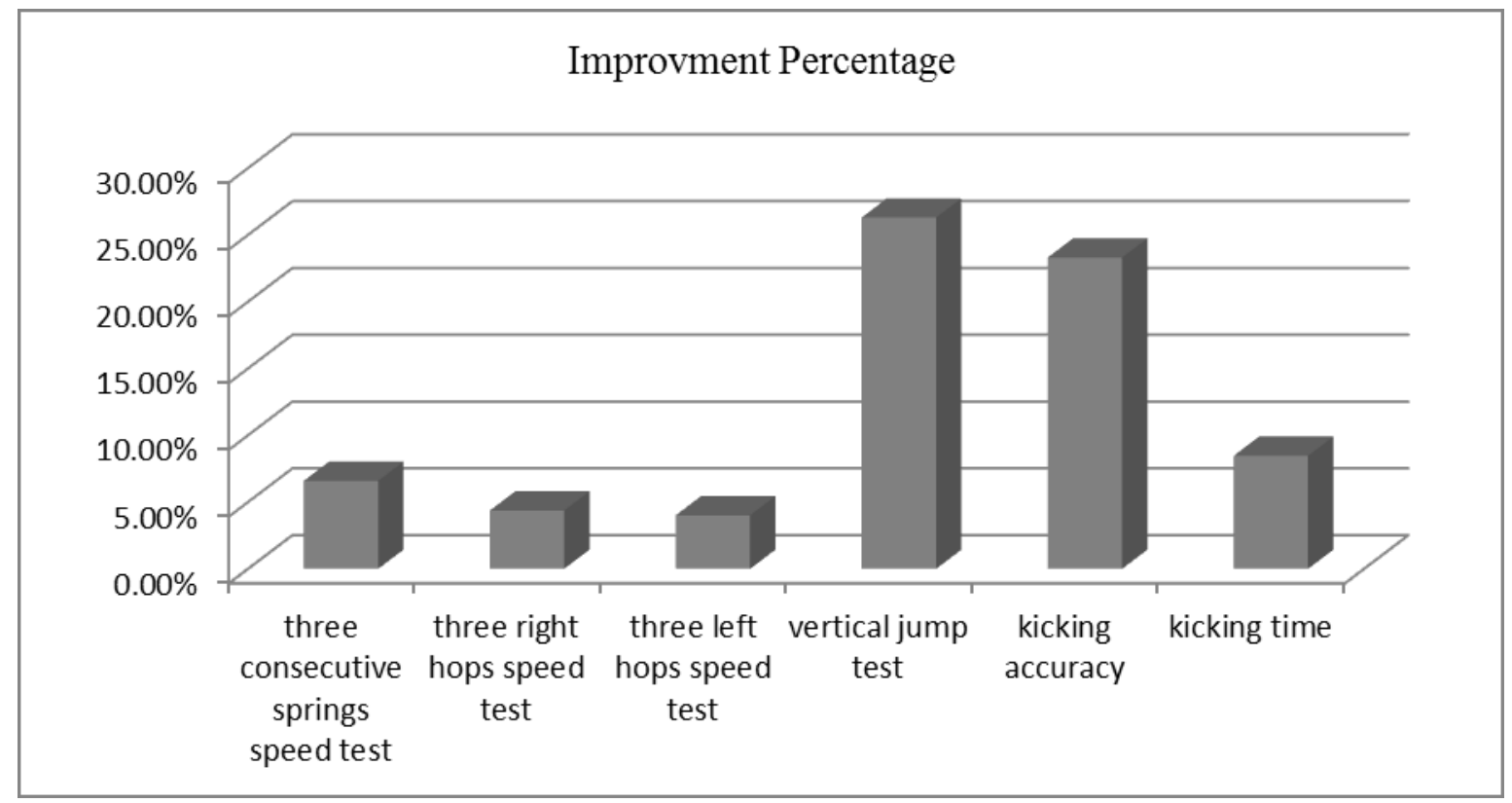

Figure 3. Improving percentage between pre-test and post-test in special strength and instep kick variables of control group

Table 4. Differences between pre-test and post-test of the kinematic variables of the control group

\begin{tabular}{|c|c|c|c|c|c|c|c|c|}
\hline \multirow[b]{2}{*}{$n=10$} & \multirow[b]{2}{*}{ Variables } & \multirow[b]{2}{*}{ skill Stages } & \multicolumn{2}{|c|}{ Pre-test } & \multicolumn{2}{|c|}{ Post test } & \multirow[b]{2}{*}{ t-value } & \multirow{2}{*}{$\begin{array}{c}\text { Improveme } \\
\text { nt } \\
\text { Percentage }\end{array}$} \\
\hline & & & Mean & $\begin{array}{c}\text { Std. } \\
\text { D }\end{array}$ & Mean & Std. D & & \\
\hline \multirow{12}{*}{$\begin{array}{l}\text { Hip } \\
\text { Joint }\end{array}$} & \multirow{3}{*}{$\begin{array}{c}\text { Angular } \\
\text { displacement }(\theta)\end{array}$} & backswing & 161.7 & 11.67 & 164.6 & 3.10 & 0.888 & $\% 1.79$ \\
\hline & & Impact & 126.18 & 2.25 & 126.63 & 2.38 & 0.387 & $\% 0.36$ \\
\hline & & Follow-up & 116.76 & 0.52 & 117.02 & 2.44 & 0.363 & $\% 0.22$ \\
\hline & \multirow{3}{*}{$\begin{array}{c}\text { Linear displacement } \\
\left(\mathrm{S}^{\mathrm{R}}\right)\end{array}$} & backswing & 0.267 & 0.02 & 0.278 & 0.04 & 0.866 & $\% 4.12$ \\
\hline & & Impact & 0.197 & 0.01 & 0.231 & 0.03 & 3.510 & $\% 17.26$ \\
\hline & & Follow-up & 0.274 & 0.02 & 0.283 & 0.02 & 3.857 & $\% 3.28$ \\
\hline & \multirow{3}{*}{ Angular velocity $(\omega)$} & backswing & 11.864 & 1.47 & 12.493 & 1.11 & 2.994 & $\% 5.30$ \\
\hline & & Impact & 0.429 & 0.02 & 0.52 & 0.06 & 4.021 & $\% 21.21$ \\
\hline & & Follow-up & 7.297 & 1.55 & 8.387 & 1.02 & 2.245 & $\% 14.94$ \\
\hline & \multirow{3}{*}{ Linear velocity $\left(\mathrm{V}^{\mathrm{R}}\right)$} & backswing & 1.295 & 0.07 & 1.333 & 0.03 & 1.667 & $\% 2.93$ \\
\hline & & Impact & 1.286 & 0.06 & 1.478 & 0.19 & 2.666 & $\% 14.93$ \\
\hline & & Follow-up & 0.73 & 0.05 & 0.754 & 0.03 & 4.609 & $\% 3.29$ \\
\hline
\end{tabular}




\begin{tabular}{|c|c|c|c|c|c|c|c|c|}
\hline & \multirow{3}{*}{$\begin{array}{l}\text { Linear acceleration } \\
\left(\mathrm{a}^{\mathrm{R}}\right)\end{array}$} & backswing & 11.864 & 0.36 & 12.13 & 0.32 & 3.392 & $\% 2.24$ \\
\hline & & Impact & 10.853 & 0.61 & 11.55 & 0.64 & 2.991 & $\% 6.42$ \\
\hline & & Follow-up & 12.18 & 0.79 & 12.77 & 1.09 & 1.796 & $\% 4.84$ \\
\hline \multirow{15}{*}{$\begin{array}{l}\text { Knee } \\
\text { Joint }\end{array}$} & \multirow{3}{*}{$\begin{array}{c}\text { Angular } \\
\text { displacement }(\theta)\end{array}$} & backswing & 65 & 2.75 & 66.3 & 3.43 & 2.623 & $\% 2.00$ \\
\hline & & Impact & 109.5 & 3.44 & 110.6 & 3.10 & 4.714 & $\% 1.00$ \\
\hline & & Follow-up & 170.8 & 2.94 & 171.5 & 2.80 & 4.582 & $\% 0.41$ \\
\hline & \multirow{3}{*}{$\begin{array}{c}\text { Linear displacement } \\
\left(\mathrm{S}^{\mathrm{R}}\right)\end{array}$} & backswing & 0.416 & 0.02 & 0.265 & 0.02 & $21.37-$ & $\% 36.30-$ \\
\hline & & Impact & 1.954 & 0.08 & 1.982 & 0.11 & 1.114 & $\% 1.43$ \\
\hline & & Follow-up & 2.588 & 0.06 & 2.616 & 0.36 & 0.267 & $\% 1.08$ \\
\hline & \multirow{3}{*}{ Angular velocity $(\omega)$} & backswing & 1.844 & 0.03 & 2.162 & 0.27 & 3.807 & $\% 17.25$ \\
\hline & & Impact & 14.36 & 0.82 & 16.1 & 2.02 & 2.904 & $\% 12.12$ \\
\hline & & Follow-up & 0.29 & 0.01 & 0.318 & 0.01 & 9.635 & $\% 9.66$ \\
\hline & \multirow{3}{*}{ Linear velocity $\left(\mathrm{V}^{\mathrm{R}}\right)$} & backswing & 4.371 & 0.21 & 4.96 & 0.43 & 4.848 & $\% 13.48$ \\
\hline & & Impact & 1.255 & 0.30 & 1.62 & 0.29 & 7.378 & $\% 29.08$ \\
\hline & & Follow-up & 2.64 & 0.32 & 2.934 & 0.25 & 2.631 & $\% 11.14$ \\
\hline & \multirow{3}{*}{$\begin{array}{l}\text { Linear acceleration } \\
\left(\mathrm{a}^{\mathrm{R}}\right)\end{array}$} & backswing & 51.84 & 0.99 & 52.575 & 1.12 & 2.258 & $\% 1.42$ \\
\hline & & Impact & 58 & 1.05 & 59.15 & 0.88 & 5.437 & $\% 1.98$ \\
\hline & & Follow-up & 33 & 0.67 & 34 & 0.67 & 6.708 & $\% 3.03$ \\
\hline \multirow{15}{*}{$\begin{array}{l}\text { Ankle } \\
\text { Joint }\end{array}$} & \multirow{3}{*}{$\begin{array}{c}\text { Angular } \\
\text { displacement }(\theta)\end{array}$} & backswing & 151 & 1.05 & 151.55 & 1.01 & 1.492 & $\% 0.36$ \\
\hline & & Impact & 127.2 & 1.23 & 129.4 & 1.51 & 8.819 & $\% 1.73$ \\
\hline & & Follow-up & 138.3 & 1.16 & 138.6 & 1.07 & 1.963 & $\% 0.22$ \\
\hline & \multirow{3}{*}{$\begin{array}{l}\text { Linear displacement } \\
\left(S^{R}\right)\end{array}$} & backswing & 0.38 & 0.01 & 0.398 & 0.01 & 6.194 & $\% 4.74$ \\
\hline & & Impact & 0.421 & 0.01 & 0.443 & 0.01 & 5.659 & $\% 5.23$ \\
\hline & & Follow-up & 0.613 & 0.01 & 0.645 & 0.01 & 8.231 & $\% 5.22$ \\
\hline & \multirow{3}{*}{ Angular velocity $(\omega)$} & backswing & 3.562 & 0.01 & 4.016 & 0.60 & 2.396 & $\% 12.75$ \\
\hline & & Impact & 2.831 & 0.47 & 3.389 & 0.38 & 6.374 & $\% 19.71$ \\
\hline & & Follow-up & 1.499 & 0.20 & 1.692 & 0.14 & 5.505 & $\% 12.88$ \\
\hline & \multirow{3}{*}{ Linear velocity $\left(\mathrm{V}^{\mathrm{R}}\right)$} & backswing & 5.14 & 0.17 & 5.63 & 0.26 & 6.133 & $\% 9.53$ \\
\hline & & Impact & 5.631 & 1.14 & 6.14 & 0.73 & 3.324 & $\% 9.04$ \\
\hline & & Follow-up & 2.709 & 0.21 & 3.202 & 0.28 & 10.400 & $\% 18.20$ \\
\hline & \multirow{3}{*}{$\begin{array}{l}\text { Linear acceleration } \\
\left(a^{\mathrm{R}}\right)\end{array}$} & backswing & 23.885 & 0.82 & 25.8 & 1.01 & 12.165 & $\% 8.02$ \\
\hline & & Impact & 125.3 & 1.77 & 127.5 & 1.18 & 7.570 & $\% 1.76$ \\
\hline & & Follow-up & 52.2 & 0.79 & 53.7 & 1.06 & 5.581 & $\% 2.87$ \\
\hline
\end{tabular}

\section{T. spreadsheet at $0.05=1.833$}

Table (4) indicates the existence of significant difference between the pre-test and post-test in kinematic variables (Angular displacement $(\theta)$-Linear displacement (SR)- Angular velocity ( $\omega$ )- Linear velocity (VR)-Linear acceleration $(\mathrm{aR}))$ of the control group for the post-test.

Table 5. Differences between post-test of the experimental group and post-test of the control group in Special Strength and instep kick

\begin{tabular}{|c|c|c|c|c|c|c|}
\hline \multirow{2}{*}{$\begin{array}{l}\mathrm{n} 1=\mathrm{n} 2=10 \\
\text { Variables }\end{array}$} & \multirow{2}{*}{ 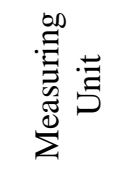 } & \multicolumn{2}{|c|}{ Post test } & \multicolumn{2}{|c|}{ Post test } & \multirow{2}{*}{ 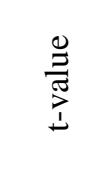 } \\
\hline & & Mean & Std. D & Mean & Std. D & \\
\hline three consecutive springs speed test & $\mathrm{m} / \mathrm{sec}$ & 2.799 & 0.02 & 2.65 & 0.02 & 13.66 \\
\hline three right hops speed test & $\mathrm{m} / \mathrm{sec}$ & 2.985 & 0.09 & 2.82 & 0.03 & 4.93 \\
\hline three left hops speed test & $\mathrm{m} / \mathrm{sec}$ & 3.01 & 0.07 & 2.79 & 0.02 & 8.06 \\
\hline vertical jump test & $\mathrm{cm}$ & 73.7 & 2.67 & 64.3 & 2.31 & 8.33 \\
\hline kicking accuracy & Degree & 4.385 & 0.02 & 3.42 & 0.20 & 15.47 \\
\hline kicking time & $\mathrm{sec}$ & 0.28 & 0.02 & 0.34 & 0.01 & 7.57 \\
\hline
\end{tabular}

T. spreadsheet at $0.05=1.833$

Table (5) indicates the existence of significant difference between post-test of the experimental group and post-test of the control group in special strength and instep kicking time and accuracy for the post-test of the experimental group. 
Table 6. Differences between post-tests of experimental group and control group in kinematic variables

\begin{tabular}{|c|c|c|c|c|c|c|c|}
\hline \multirow{2}{*}{$\mathrm{n} 1=\mathrm{n} 2=10$} & \multirow{2}{*}{ Variables } & \multirow{2}{*}{ skill Stages } & \multicolumn{2}{|c|}{ experimental group } & \multicolumn{2}{|c|}{ control group } & \multirow{2}{*}{ t-value } \\
\hline & & & Mean & Std. D & Mean & Std. D & \\
\hline \multirow{15}{*}{ Hip Joint } & \multirow{3}{*}{$\begin{array}{c}\text { Angular } \\
\text { displacement }(\theta)\end{array}$} & backswing & 175.7 & 1.34 & 164.6 & 3.10 & 12.008 \\
\hline & & Impact & 131.13 & 0.74 & 126.63 & 2.38 & 5.708 \\
\hline & & Follow-up & 118.64 & 0.13 & 117.02 & 2.44 & 2.143 \\
\hline & \multirow{3}{*}{$\begin{array}{c}\text { Linear } \\
\text { displacement }\left(\mathrm{S}^{\mathrm{R}}\right)\end{array}$} & backswing & 0.356 & 0.02 & 0.278 & 0.04 & 6.049 \\
\hline & & Impact & 0.269 & 0.01 & 0.231 & 0.03 & 5.338 \\
\hline & & Follow-up & 0.371 & 0.01 & 0.283 & 0.02 & 12.943 \\
\hline & \multirow{3}{*}{$\begin{array}{l}\text { Angular velocity } \\
(\omega)\end{array}$} & backswing & 18.95 & 0.01 & 12.493 & 1.11 & 18.503 \\
\hline & & Impact & 0.62 & 0.01 & 0.52 & 0.06 & 4.74342 \\
\hline & & Follow-up & 9.221 & 0.01 & 8.387 & 1.02 & 2.569 \\
\hline & \multirow{3}{*}{$\begin{array}{l}\text { Linear velocity } \\
\left(\mathrm{V}^{\mathrm{R}}\right)\end{array}$} & backswing & 1.828 & 0.01 & 1.333 & 0.03 & 46.842 \\
\hline & & Impact & 1.821 & 0.01 & 1.478 & 0.19 & 5.585 \\
\hline & & Follow-up & 1.109 & 0.15 & 0.754 & 0.03 & 8.244 \\
\hline & \multirow{3}{*}{$\begin{array}{c}\text { Linear } \\
\text { acceleration }\left(\mathrm{a}^{\mathrm{R}}\right)\end{array}$} & backswing & 13.942 & 0.02 & 12.13 & 0.32 & 17.831 \\
\hline & & Impact & 13.757 & 0.15 & 11.55 & 0.64 & 11.875 \\
\hline & & Follow-up & 15.8 & 0.09 & 12.77 & 1.09 & 8.498 \\
\hline \multirow{15}{*}{ Knee Joint } & \multirow{3}{*}{$\begin{array}{c}\text { Angular } \\
\text { displacement }(\theta)\end{array}$} & backswing & 68.4 & 1.07 & 66.3 & 3.43 & 2.214 \\
\hline & & Impact & 112.8 & 0.79 & 110.6 & 3.10 & 2.369 \\
\hline & & Follow-up & 178.5 & 1.08 & 171.5 & 2.80 & 7.333 \\
\hline & \multirow{3}{*}{$\begin{array}{c}\text { Linear } \\
\text { displacement }\left(S^{R}\right)\end{array}$} & backswing & 0.318 & 0.02 & 0.265 & 0.02 & 4.791 \\
\hline & & Impact & 2.03 & 0.04 & 1.982 & 0.11 & 1.841 \\
\hline & & Follow-up & 2.725 & 0.02 & 2.616 & 0.36 & 1 \\
\hline & \multirow{3}{*}{$\begin{array}{l}\text { Angular velocity } \\
(\omega)\end{array}$} & backswing & 2.461 & 0.07 & 2.162 & 0.27 & 3.542 \\
\hline & & Impact & 19.195 & 0.63 & 16.1 & 2.02 & 3.895 \\
\hline & & Follow-up & 0.347 & 0.01 & 0.318 & 0.01 & 5.513 \\
\hline & \multirow{3}{*}{$\begin{array}{l}\text { Linear velocity } \\
\left(\mathrm{V}^{\mathrm{R}}\right)\end{array}$} & backswing & 5.638 & 0.15 & 4.96 & 0.43 & 6.137 \\
\hline & & Impact & 2.638 & 0.20 & 1.62 & 0.29 & 12.501 \\
\hline & & Follow-up & 3.223 & 0.03 & 2.934 & 0.25 & 3.610 \\
\hline & \multirow{3}{*}{$\begin{array}{c}\text { Linear } \\
\text { acceleration }\left(\mathrm{a}^{\mathrm{R}}\right)\end{array}$} & backswing & 55.505 & 0.28 & 52.575 & 1.12 & 7.824 \\
\hline & & Impact & 61.1 & 1.10 & 59.15 & 0.88 & 6.090 \\
\hline & & Follow-up & 35.5 & 1.43 & 34 & 0.67 & 3.737 \\
\hline \multirow{15}{*}{ Ankle Joint } & \multirow{3}{*}{$\begin{array}{c}\text { Angular } \\
\text { displacement }(\theta)\end{array}$} & backswing & 152.5 & 0.97 & 151.55 & 1.01 & 2.69 \\
\hline & & Impact & 133.6 & 1.26 & 129.4 & 1.51 & 7.875 \\
\hline & & Follow-up & 139.6 & 0.84 & 138.6 & 1.07 & 3.354 \\
\hline & \multirow{3}{*}{$\begin{array}{c}\text { Linear } \\
\text { displacement }\left(S^{R}\right)\end{array}$} & backswing & 0.423 & 0.01 & 0.398 & 0.01 & 6.228 \\
\hline & & Impact & 0.46 & 0.02 & 0.443 & 0.01 & 2.939 \\
\hline & & Follow-up & 0.686 & 0.02 & 0.645 & 0.01 & 9.461 \\
\hline & \multirow{3}{*}{$\begin{array}{l}\text { Angular velocity } \\
\qquad(\omega)\end{array}$} & backswing & 4.527 & 0.76 & 4.016 & 0.60 & 3.812 \\
\hline & & Impact & 4.693 & 0.07 & 3.389 & 0.38 & 9.887 \\
\hline & & Follow-up & 1.953 & 0.02 & 1.692 & 0.14 & 5.591 \\
\hline & & backswing & 6.485 & 0.18 & 5.63 & 0.26 & 11.409 \\
\hline & Linear velocity & Impact & 8.959 & 0.02 & 6.14 & 0.73 & 12.178 \\
\hline & & Follow-up & 3.839 & 0.06 & 3.202 & 0.28 & 7.455 \\
\hline & & backswing & 28.7 & 0.82 & 25.8 & 1.01 & 7.010 \\
\hline & Linear & Impact & 130.5 & 0.71 & 127.5 & 1.18 & 7.115 \\
\hline & acceleration $\left(\mathrm{a}^{\mathrm{N}}\right)$ & Follow-up & 54.9 & 0.88 & 53.7 & 1.06 & 4.129 \\
\hline
\end{tabular}

T. spreadsheet at $0.05=1.833$

Table (6) indicates the existence of significant difference between post-test of the experimental group and post-test of the control group in kinematic variables (Angular displacement $(\theta)$-Linear displacement (SR)- Angular velocity ( $\omega$ )Linear velocity (VR)-Linear acceleration $(\mathrm{aR})$ ) for the post-test of the experimental group.

\section{Discussion}

As table (1) indicated the existence of significant differences between the pre-test and the post-test of the experimental group at (0.05) level for the post-test in the special strength variables including (three consecutive springs speed - three right hops speed- three left hops speed- vertical jump) tests. The highest rate of change was for the vertical jump test with $16 \%$ percentage while, the lowest rate of change was for the three right hops speed test with $9.74 \%$ percentage.

In the instep kick level including (kicking accuracy - kicking time), the highest rate of change was for the kicking accuracy test with $58.30 \%$ percentage while , the least rate of change was for the kicking time test with $26.70 \%$ percentage that indicates the significant improvement in these variable in the post-test. This improvement is due to the training program containing special exercises to develop the special strength, clarifying that selecting the proper exercises allows coaches to develop the players physical and skill characteristics as well as these exercises took into account the correlation between the physical and skill sides considering the performance nature in soccer.

This agree with what (Burnett ,2004; Chek , 2001 ; Comana , 2004 ; Gaines ,2003 ; Jones ,2007; Schmitz , 2003 ; González, et. al. 2012) have mentioned clarifying that the special strength development had contributed in significantly increasing hip and legs muscles strength. The researcher also believes that the instep kick requires a certain 
amount of strength to perform the kinetic performance of the kicking process besides organizing, guiding and distributing strength to produce the needed kinetic energy and successful kinetic performance.

According to the researcher this improvement in the instep kick represented by kicking accuracy and kicking time, is due to players ability on employing the gained strength in lower limb muscles participating in the instep kick in performing good kinetic transmission of the gained strength from leg to ankle to generate large quantity of movement in the instep to transmit to the soccer. Where, the kinetic resistance exercises associated with the performance shape and applied on the experimental group to improve the power and kinetic transmission for the muscular function to the muscular groups working in the instep kick.

The results in table (2) indicated the existence of significant differences between the pre-test and post-test of the experimental group at (0.05) level for the post-test in the kinematic variables including linear and angular displacement of the hip joint, angular velocity, Linear velocity and Linear acceleration. This improvement is significantly obvious during the movement preliminary stage which allows the player to increase the scale of the leg back swing and thus generate greater strength during primary of the instep kick. There is also an obvious improvement in the post-test over the pre-test in linear and angular displacement, Linear and angular velocity and Linear acceleration of the hip joint which indicates the transmission of large quantity of energy from hip to knee.

The linear and angular displacement, Linear and angular velocity and Linear acceleration of the ankle also showed an obvious improvement whereas, the largest angular displacement was during the preliminary stage while the largest Linear velocity was during the primary stage having the ankle speed greater than the hip and knee speed which indicate the transmission of the kinetic energy from hip to the knee to ankle which is considered the fastest point with larger linear acceleration than the hip and knee during this stage.

The researcher due the existence of significant differences for the post test to the training program that involves different exercises to develop the lower limb special strength and the instep kick performance level. These exercises concentrated on binding the physical characteristics with the performance nature through the speed and the accuracy needed by the performance. These results agrees with what (De Proft et, al., 1988 \& Manolopoulos et. al.,2013) have mentioned clarifying that the speed of knee flexion and expansion is one of the major factors that influence soccer kicking skill.

As table (3) indicated the existence of significant differences between the pre-test and the post-test of the control group at $(0.05)$ level for the post-test in the special strength variables. The highest rate of change was for the vertical jump test with $26.30 \%$ percentage while, the lowest rate of change was for the three right hops speed test with $4.01 \%$ percentage. In the instep kick level, the highest rate of change was for the kicking accuracy test with $23.31 \%$ percentage while, the lowest rate of change was for the kicking time test with $\% 8.47$ percentage that indicates a significant improvement in these variable in the post-test.

The results in table (4) indicated the existence of significant differences between the pre-test and post-test of the control group at (0.05) level for the post-test in the kinematic variables including angular displacement of the hip joint, angular velocity, linear velocity and linear acceleration. There is also an obvious significantly improvement for the post-test in the linear and angular displacement, Linear and angular velocity and Linear acceleration of the knee joint which , indicates the transmission of large quantity of energy from hip to knee. Also there is also an obvious significantly improvement for the post-test in the linear and angular displacement, Linear and angular velocity and Linear acceleration of the ankle joint.

The researcher due this improvement to the traditional training program that utilized the training methods and steps on the physical and skill characteristics, assuring that any scientifically rated training program must lead to an improvement in the physical and skill performance level except that the amount of improvement is the criterion between the two programs.

Table (5) indicated the existence of significant differences between the post-test of the experimental group and the posttest of the control group at (0.05) level for the post-test of the experimental group in the special strength variables. The highest rate of change was for the three consecutive springs speed test with $\% 13.26$ percentage while, the lowest rate of change was for the three right hops speed test with $\% 4.93$ percentage.

In the instep kick level, the highest rate of change was for the kicking accuracy test with $\% 15.47$ percentage while, the lowest rate of change was for the kicking time test with \%7.57 percentage that indicates the obvious significant improvement in these variable in the post-test. This improvement in the kicking accuracy and time is due to the training program containing special exercises to develop the special strength, taking into account the correlation between the physical and skill sides considering the performance nature in soccer.

The researcher believes that through executing the kinetic resistance training program, exercises is performed with the maximum speed to commensurate with the special action of the soccer kinetic performance, which agree with what (Haghighi et.al., 2012; Harries et.al., 2012; Brown , 2000 ; Manolopoulos et. al., 2015 \& Schmitz, 2003) have mentioned clarifying that most actions dedicated to the kinetic performance are performed at high speed and increasing the strength at high speed of the movement should lead to sportive performance increasing. The strength also should be organized, guided and distributed to produce the needed kinetic energy and have the successful kinetic performance.

The results in table (6) indicated the existence of significant differences between the post-test of the experimental group 
and the post-test of the control group at $(0.05)$ level for the post-test of the experimental group in the kinematic variables under consideration. There is an obvious significantly improvement for the post-test in the linear and angular displacement, Linear and angular velocity and Linear acceleration of the hip joint, which is obvious during the preliminary stage and allows the player to increase the scale of the leg back swing and thus generate greater strength during the primary stage. There is also an obvious improvement in the post-test over the pre-test in linear and angular displacement, Linear and angular velocity and Linear acceleration of the hip joint achieving the highest and angular displacement and angular velocity of the hip joint in the primary stage. While, the linear velocity of the knee joint was greater than the Linear velocity of the hip joint of the experimental group indicating the transmission of large quantity of energy from hip to knee.

The experimental group has achieved significant superiority over the control group in linear and angular displacement, Linear velocity and Linear acceleration of the ankle. The highest angular displacement was in the preliminary stage and the highest linear velocity was in the primary stage whereas the ankle speed was greater than the hip and knee during this stage indicating the transmission of kinetic energy from hip to knee to ankle to be the fastest point during the primary stage. The ankle also gained the highest linear acceleration during the primary stage a lot more exceeding the hip and knee.

Table (6) indicates that linear velocity outcome average during performance stages of the instep, the highest speed outcome was at the moment of impact with the ball and the lowest speed outcome was at the maximal back swing. Considering the knee, the the highest speed outcome was at the maximal back swing and the lowest speed outcome was at the moment of impact with the ball and finally for the hip, the the highest speed outcome was at the maximal back swing and the lowest speed outcome was at the maximal forward swing.

These previous results agree with what (Masuda et.al., 2005 \& Manolopoulos et.al.,2006) have mentioned clarifying that the instep kick is one of the open kinetic chain skills that requires good kinetic transmission to produce the largest possible quantity of speed and strength though the movement of the chain parts to the end of the chain.

This also agrees with what (Barfield ,1998 \& Manolopoulos et.al., 2013) have reached clarifying that the muscles activities levels was higher during the kicking whereas the maximum hip and knee muscles activities happened through the final stage which increase one more time before the moment of impact with the ball.

\section{Conclusions}

Believing of the important role and impact of kinetic resistance trainings on enhancing soccer players abilities specially soccer juniors concerning the special strength and effective kinematic variables in instep kick, the researcher have introduced this research. Through this research, the researcher investigated the effect of kinetic resistance trainings and technique on special strength level and effective kinematic variables in instep kick for soccer juniors. The experiment results indicated that the kinetic resistance training and technique have achieved positive change in the special strength level, in the instep kick accuracy and time and kinematic variables affecting the instep kick of soccer juniors. From these results the researcher strongly recommended that coaches need to pay attention to developing special strength through having kinetic resistance exercises and good technique as an essential part of soccer juniors special strength training programs. The researcher also recommended to depend on kinematic variables affecting performance to recognize the improvement amount in performance as a result of kinetic resistance exercises and technique.

\section{References}

Barfield, B. (1998). The Kinetic of Kicking in Soccer. Clinicsin Sports Medicine,17(4),711-728.

Brown, L. (2000). Isokinetics In Human Performance. (1 ${ }^{\text {st }}$ ed.). Human Kinetics, USA.

Burnett, A. (2004). The Biomechanics of Jumping. [Online] Available: www.coachsinfo.com. (October, 15,2015).

Cabri, J., De Proft, E., Dufour, W. \& Clarys, J. (1988). The Relation between Muscular Strength and Kick Performance. Science and Football. Eds: Reilly, T., Lees, A., Davids, K. and Murphy, W. London: E \& FN Spon. 186193.

Chek, P. (2001). Big Bang Exercise. IDEA Fitness Edge, 8-10. [Online] Available: http://www.ideafit.com/fitnessproducts/big-bang-exercises. (October, 1, 2015).

Comana, F. (2004). Function Training For Sports. ( $1^{\text {st }}$ ed.). Human Kinetics. Champaign IL, England.

De Proft, E., Cabri, J, Dufour, W, Clarys, J. (1988). Strength Training and Kick Performance in Soccer Players. Reilly T, Lees A, Davids K, Murphy WJ, eds. Science and football. London: E \& FN Spon, 109-113.

El-Berawe, E. \& Shady. A. (2013). Effectiveness of Special Strength Training on Some Physical and Kinetic Parameters Affecting Instep Kick For Soccer Juniors. Theories \& Applications the International Edition, 3(2), 146-155.

Evans, M. (1997). Endurance Athlete'S Edge. Human Kinetics, USA.

Gaines, S. (2003). Benefits and Limitations of Quality Exercise, Vertex Fitness . NESTA, USA.

Ghigiarelli, J., Nagle, E., Gross, F., Robertson, R. , Irrgang, J. , \& Myslinski, T. (2009). The effects of a 7-week heavy elastic band and weight chain program on upper-body strength and upper-body power in a sample of division 1-AA football players. The Journal of Strength \& Conditioning Research, 23(3), 756-764. 
González-Badillo, J. J., Pareja-Blanco, F., Rodríguez-Rosell, D., Abad-Herencia, J. L., del Ojo-López, J. J., \& SánchezMedina, L. (2015). Effects of Velocity-Based Resistance Training on Young Soccer Players of Different Ages. The Journal of Strength \& Conditioning Research, 29(5), 1329-1338.

Haghighi, A., Moghadasi, M., Nikseresht, A., Torkfar, A \&Haghighi, M. (2012). Effects Of Plyometric Versus Resistance Training On Sprint And Skill Performance In Young Soccer Players. European Journal of Experimental Biology, 2 (6),2348-235.

Harries, S., Lubans, D. \& Callister, R. (2012). Resistance Training To Improve Power And Sports Performance In Adolescent Athletes: A Systematic Review And Meta-Analysis. Journal of Science and Medicine in Sport ,15, 532540 .

Inoue, K. , Nunome, H. , Sterzing, T., Shinkai, H. \& Ikegami, Y. (2012). Kinetic Analysis of the Support Leg in Soccer Instep Kicking. 30th Annual Conference of Biomechanics in Sports, Melbourne, 1(1).

Jones, R. (2007). Functional Training. Introduction. Reebo Santana, Jose Carlos Uni. USA, 9-15. [Online] Available: http://ronjones.org/Handouts/FT1-Intro\&Assessment.pdf

Keshk, M. \& Elbosaty, A. (2000). Skill and Tactic Preparation Foundations In Soccer (Juniors-Adults). (1 ${ }^{\text {st }}$ ed.). Mansoura.

Manolopoulos, E., Gissis, I, Galazoulas, C., Manolopoulos, E., Patikas, D., Gollhofer, A. \&Kotzamanidis, C. (2016). The Effect of Combined Sensorimotor-Resistance Training On Strength, Balance and Jumping Performance of Soccer Players. In Journal of Strength \& Conditioning Research. 30 (1): 53-9.

Manolopoulos, E., Katis, A., Manolopoulos, K., Kalapotharakos, V. \& Kellis, E. (2013). Effects of a 10-Week Resistance Exercise Program on Soccer Kick Biomechanics and Muscle Strength. Journal of Strength \& Conditioning Research, 27(12), 3391-3401.

Manolopoulos, E., Papadopoulos, C. \& Kellis, E. (2006). Effects of Combined Strength And Kick Coordination Training On Soccer Kick Kinetic in Amateur Players. Scandinavian Journal of Medicine \& Science in Sports.16(2), 102-110.

Masuda, K., Kikuhara, N., Demura, S., Katsuta, S. \& Yamanaka, K. (2005). Relationship between Muscle Strength In Various Isokinetic Movements And Kick Performance Among Soccer Players. J. sports med. Phys. Fitness, 45, 44-52.

Moran, G. \& Glynn, G. (2000). Dynamics Of Strength Trining And Conditioning. (3rd ed.). Wcb Mc Grow, Hill, New York, USA.

Noguchi, T., Demura, S. \& Nagasawa, Y. (2012). Relationship between Ball Kick Velocity and Leg Strength: A Comparison between Soccer Players and Other Athletes. SciRes 2(3),95-98.

Perrin, D. (1993).Isokinetic Exercise and assessment . (1 ${ }^{\text {st }}$ ed.). Human Kinetics, USA.

Reilly, T. \& Williams, A. (2003). Science and Soccer. ( $2^{\text {nd }}$ ed.). Taylor \& Francis e-Library.

Roberts, S. \& Weider, B. (1994). Strength and Weight Training For Young Athletes Contemporary, ( $1^{\text {st }}$ ed.). Book 5 Inc Publisher, Chicago USA.

Schmitz, D. (2003). Quality Training Pyramids. New Truer High School, Kinetic Wellness Department, USA.

Resistance Bands. [Online] Available:http://myosource.com/kinetic-bands-leg-resistance-exercise-bands/.(October, 2, 2015). 